\title{
LES ERREURS DE DÉTERMINANTS CHEZ LES ÉTUDIANTS EN FRANÇAIS LANGUE ÉTRANGÈRE: UNE PERSPECTIVE MICROTEXTUELLE
}

\begin{abstract}
A bst r act . Jespersen Janine, Les erreurs de déterminants chez les étudiants en français langue étrangère: une perspective microtextuelle [Errors in the use of articles made by the students of French: a microtextual perspective]. Studia Romanica Posnaniensia, Adam Mickiewicz University Press, Poznań, vol. XXV/XXVI: 2000, pp. 167-181, ISBN 83-232-0965-0, ISSN 0137-2475.
\end{abstract}

The goal of this paper is to provide teachers of French with the origin of main errors made by Slavic students in the use of articles. In the languages of these students there are no articles.

Of particular interest are binominal structures, for example: text analysis, (l'analyse de texte) vs analysis of the text, (l'analyse du texte). In the first example an entity is subcategorised as a generic entity; in the second example this expression is used as the noun of a process: the text was read, discussed, explored The analysis of the text

Constructions of this type are often used in order to provide summaries and introduce abstractions in scientific and academic texts.

I shall apply the theory of Alain Berrendonner to these structures and I shall suggest some ways of correcting errors in this area.

\section{PRÉLIMINAIRES}

Contrairement à ce que le titre promet, la majeure partie de cet article est réservée à la description de la structure interne du syntagme binominal, en particulier à une étude sur pièce du fonctionnement de N2, qui détermine deux catégories de SN: les spécifieurs $\rightarrow$ l'analyse du texte et les catégorisateurs $\rightarrow$ l'analyse de texte. Toutefois c'est l'analyse des erreurs qui documente notre approche méthodologique. Le fonctionnement textuel de ces deux constructions se distingue nettement. Alors que [N1 DU N2] fonctionne souvent comme une anaphore résomptive, [ce N1 DU N2], condensant un discours antérieur, [N1 DE ØN2] effectue un décrochement énonciatif qui permet de passer de l'individuel au général et cette «abstraction» signale souvent le passage de l'informatif-descriptif-narratif à l'argumentatif. Cependant ce n'est pas ce fonctionnement textuel qui nous retiendra aujourd'hui, mais plutôt la combinatoire interne du syntagme. 


\section{CORPUS D'ÉTUDE}

La présente étude s'inscrit dans un projet de recherche appliquée à l'enseignement $\mathrm{du}$ français, que je mène avec trois collègues du département de romanistique de l'Université de Lomonossov à Moscou. Ce projet de langues en contraste est financé par le fonds de la recherche scientifique suisse, (FNRS) et a pour but de fournir des outils pédagogiques aux enseignants de français langue étrangère.

Notre corpus d'étude comprend des textes produits par les étudiants slavophones à l'examen de langue, auquel tout étudiant étranger est soumis pour être admis à l'Université de Genève, et des textes argumentatifs produits par des étudiants avancés, préparant un diplôme d'études françaises et ayant reçu un enseignement sur l'usage des déterminants.

Par leur pouvoir de synthèse et de conceptualisation, les constructions à N2 spécifieur et sous-catégorisateur représentent la grande majorité des syntagmes binominaux. Beaucoup de titres d'articles de presse en fournissent d'ailleurs la preuve:

1. Russie: Incendies de forêt

Près de 25000 hectares de forêt sont actuellement en feu dans l'Extrême-Orient russe

L'information en 10 lignes est annoncée par deux SN: $\varnothing$ Russie $=[1 \mathrm{SN}$ simple]/Incendies de forêt: [1 SN complexe: N2 = sous-catégorisateur].

Cette nominalisation est à la fois résomptive et conceptuelle, puisque, comme le singulier de forêt l'indique, elle ne fait pas que de résumer un contenu propositionnel antérieur, mais elle reclasse l'événement particulier en catégorie abstraite incendies de forêt, le faisant entrer ainsi dans la rubrique des divers types d'embrasement: incendies de baraquements/ feux de cave/de grenier.

\section{SPÉCIFIEURS VS SOUS-CATÉGORISATEURS}

C'est donc au vu de ce que nous a fourni une analyse de quelque 500 copies, que nous avons décidé de cibler notre recherche sur les syntagmes nominaux complexes du type:

le chien du berger vs un chien de berger l'enseignement du français vs un enseignement de français

A cela trois raisons:

a) Les erreurs sont nombreuses sur ces formations, et elles sont plus tributaires d'un enseignement antérieur mal informé ou lacunaire que des modèles de la langue maternelle, souvent inexistants, puisque les langues slaves n'ont pas de déterminants.

b) L'importance des binominaux dans les activités de réduction et commentaire de discours.

c) Le manque d'outils pédagogiques, alors que de nouvelles hypothèses de la linguistique fondamentale foisonnent. Mentionnons particulièrement Wilmet, (1986), Karolak, (1989), Berrendonner, (1995). 
Parmi ce luxe de descriptions théoriques nous nous sommes appuyés plus spécialement sur les modélisations fournies par A. Berrendonner [1995: 9-39]. Voici comment, dans une perspective pragmatico-cognitive, il analyse les constructions binominales [N1] DU [N2], [N1 DE N2].

L'opérateur de repérage [DE] peut prendre pour argument soit un objet-type, soit un objet du monde réel.

Reprenons l'opposition illustrée par Bally:

2a) le chien du berger vs $2 b$ ) le chien de berger

a) En (2a), on associe au nom-tềte [Nldéf] un objet extensionnel, pensé comme existant in re, et désignant un individu précis d'une catégorie. Par exemple un berger de connaissance partagée, le berger Achille. Cette saisie situe le premier référent, jugé mal défini, par rapport à un deuxième objet bien repéré. Bally nommait [N2déf] un localisateur:

Le prédicat nominal «chien» prend pour argument un exemplaire concret et déjà repéré d'une autre catégorie, qu'il est le seul à posséder. Ce complément-déterminant, plus stable, facilite la désignation de N1. On a alors deux objets de pensée, $(1+1=2)$ comme le manifeste la présence d'un article devant $\mathrm{N} 2$.

En (2b), [ØN2] est pris dans sa valeur de catégorisateur générique, on crée ainsi une sous-catégorie dans l'espèce «chien», si bien que l'on a un seul objet de pensée complexe, c'est-à-dire plus riche en composantes catégorielles. $(1 \times 1=1)$. «Berger» n'est pas actualisé, il est pris dans sa valeur de concept et non dans sa valeur référentielle. Le syntagme binominal ne représente qu'un seul objet de pensée, donc l'article intérieur ne variera pas, quelle que soit la saisie du SN, qui sera marquée uniquement par l'article devant N1. Le SN binominal réagira comme un nom composé. Comparez:

3.1. J'ai acheté une voiture de sport

3.2. J'adore les voitures de sport

3.3. Ce garage est spécialisé en voitures de sport

La fonction sémantique d'un $[\mathrm{N} \emptyset]$ consiste à distinguer une catégorie d'une autre 1 . Il n'a pas de valeur référentielle mais «différentielle».

Le spécifieur est, comme son nom l'indique, le filtre le plus fin, puisqu'il individualise complétement le référent, de sorte qu'il n'y a qu'un seul objet correspondant à cette désignation, d'où singularité du référent, donc article défini.

${ }^{1}$ Un SN avec article zéro est donné dans sa fonction référentielle uniquement et non pas dans sa fonction d'actualisateur. Cette distinction entre actuel/virtuel est également à l'oeuvre dans les constructions appositives et attributives:

a) Son père, architecte renommé, a cependant des ennuis financiers/ Son père,l'architecte municipal, a des ennuis financiers.

b) Son père est architectelun architecte renommé.

Dans ces constructions la présence ou l'absence d'article devant le nom indique soit un titre ou une fonction, (entité notionnelle) soit un individu remplissant cette fonction, (entité singulière). 


\section{SPÉCIFIEUR ET GÉNÉRICITÉ}

Mais une difficulté surgit lorsque [N2déf] indique - conformément à la double valeur «singulière» et générique que l'article assigne au référent - un type.

4. Dans nos régions le chien du berger n'est pas un chien de berger, mais un lévrier!

Conséquence pratique: $[\mathrm{N} 2 \varnothing]$ pointe toujours sur un type, (valeur intensionnelle), mais l'inverse n'est pas vrai: [N2déf] peut tout aussi bien représenter un type: le marché de l'art, les droits de l'homme, qu'un objet extensionnel, pris dans la réalité $^{2}$ le prix du tableau. Seul le cotexte permettra d'attribuer une valeur singulative ou générique au syntagme.

D'autre part, même lorsque le spécifieur a une valeur générique, bien qu'on ressente une analogie entre la généricité et la catégorisation, il n'en reste pas moins qu'entre chien du berger et chien de berger, le référent est identifié différemment, même s'il est identique dans les deux cas. Le plus souvent d'ailleurs il est différent, comme le manifeste l'exemple (4).

J'emprunte l'exemple (5) et son commentaire très éclairant à Berrendonner [1995:20-21].

5a) Les maladies de femme vs 5 b) Les maladies de la femme

[En 4 et en 5] «le syntagme complément peut être interprété comme un nom de type. Mais selon la nature du prédéterminant le repérage par rapport au type désigné par [de SN] remplit deux fonctions différentes: l'article zéro lui confère le rôle de sous-catégorisateur, et l'article défini, un rôle de spécifieur: (5a) ouvre une rubrique dans une typologie des [maladies] tandis que (5b) ne fait que localiser une douleur catégoriellement indifférenciée. Il se peut que le référent soit à peu près le même dans les deux cas, mais il est atteint à travers deux représentations logico-sémantiques radicalement différentes. Le plus souvent d'ailleurs, il est aisé de constater que le référent n'est pas le même. (Ainsi un homme peut avoir des maladies de femme - la migraine, par exemple a la réputation d'être telle; il ne saurait, en revanche, souffrir des maladies de la femme)».

Dans les exemples (4) et (5) les syntagmes prépositionnels adnominaux ont une valeur générique. Dans l'exemple (6) qui m'a été fourni par un participant au colloque, s'interrogeant sur deux formulations en concurrence,

6a) dictionnaire de la langue française vs $6 \mathrm{~b}$ ) dictionnaire de langue française

l'article défini devant N2 pointe une entité «singulière», mais étant donné l'unicité du référent, il y a bien identité référentielle et sémantique entre les deux constructions, mais différence énonciative.

${ }^{2}$ Il s'agit aussi d'entités, mais pensées comme existant in re, saisies dans leur valeur ,,singulière”, tandis que les types, valeur intensionnelle, subsistent in intellectu, les entités qu'ils représentent ne sont pas actualisées et sont prises dans leur acception générique. 
Nous avons une parfaite illustration de cette différence dans l'édition $1988 \mathrm{du}$ Petit Robert. La page de garde annonce:

LE PETIT ROBERT 1 par Paul Robert

DICTIONNAIRE alphabétique et analogique de LA LANGUE FRANÇAISE, rédaction dirigée par A. Rey et J. Rey-Debove

La page connexe de gauche liste les ouvrages édités par les Dictionnaires LE ROBERT sous trois rubriques:

I. DICTIONNAIRES DE LANGUE, sous laquelle sont regroupés: le dictionnaire de la langue française/le dictionnaire $d u$ français primordial/le dictionnaire de la langue des sports.

II. DICTIONNAIRES DE NOMS PROPRES, qui regroupe le dictionnaire des noms propres/le dictionnaire universel de la peinture

III. DICTIONNAIRES BILINGUES qui comprend des titres tels que: le dictionnaires français-anglais/english-french ......(les italiques sont de nous).

Ainsi «le dictionnaire de la langue française» désigne un ouvrage consacré à la langue française, objet extensionnel, considérée comme une entité autonome. Il fait partie de la catégorie générale: «dictionnaires». Quant au syntagme «dictionnaire de $\phi$ langue française», il sous-catégorise deux fois l'entité «dictionnaire», premièrement dans la typologie des dictionnaires, en tant que «dictionnaire de langue» et deuxièmement dans la sous-rubrique des dictionnaire de langue, en tant que «dictionnaire de langue française». Ainsi, les deux expansions ont une valeur «différentielle» servant à désigner un type d'objet par opposition à d'autres types.

$$
\begin{gathered}
\text { N2 } \varnothing \rightarrow \text { Type } \\
\text { N2déf } \rightarrow \text { Individu } \\
\searrow \text { Type }
\end{gathered}
$$

\section{LES PROCÉDÉS DE NOMINALISATION}

A partir de 500 exemples tirés de manuels de médecine homéopatique, L. Benetti [1995: 14-15], a pu établir un tableau indicatif des fréquences d'emploi: elle relève $53,3 \%$ de spécifieurs - le taux le plus fort - pour $39,3 \%$ de catégorisateurs. Ce qui représente un total de $92,6 \%$ entre les deux combinaisons, les autres formations étant négligeables.

Ce constat rejoint mes observations, les spécifieurs sont de loin les plus représentés dans les écrits académiques, où ils constituent des allomorphes de prédicats verbaux et se prêtent à des reprises anaphoriques résomptives: les dires des touristes cette analyse du climat politique .......

On notera également qu'environ $80 \%$ des nominalisations déverbales que nous avons relevées dans la presse ont un $\mathrm{N} 2$ complément d'objet du prédicat (déverbaux objectifs). En voici quelques-unes relevées au hasard: 
7.1. La destruction de l'immeuble

7.2. Le renouvellement des cadres

7.3. Le rejet de la loi

7.4. La création d'un centre d'excellence

Les déverbaux subjectifs (N2 représentant le sujet du prédicat) sont le plus souvent issus de verbes dicendi:

8. Les déclarations de l'employé licencié

Les syntagmes déverbaux ont généralement la même construction directe ou prépositionnelle que le verbe:

9.1. Enseigner $l e$ français $\rightarrow$ L'enseignement $d u$ français

9.2. Maîtriser l'anglais $\rightarrow$ la maîtrise de l'anglais

9.3. S'abonner aux «Cahiers de grammaire» $\rightarrow$ l'abonnement aux «Cahiers de grammaire»

9.4. Changer de style $\rightarrow$ le changement de style

9.5. Manquer de légitimité $\rightarrow$ le manque de légitimité

9.6. Etre privé de livres $\rightarrow$ la privation de livres

De [9.4. à 9.6.] il y a effacement de l'article partitif ou de l'indéfini pluriel sous l'effet de l'haplologie, cf.7 infra:

Comme ces exemples le montrent, lors de la transposition de l'énoncé verbal au $\mathrm{SN}$, le déterminant choisi (article défini, indéfini, partitif) est maintenu, toutefois les règles de contraction et d'élision des articles définis et indéfinis après la préposition [DE] compliquent à plaisir l'utilisation des déterminants.

Utiliser la voiture $\rightarrow$ l'utilisation de la voiture

Utiliser de la colle $\rightarrow$ l'utilisation de colle

Ces rappels me paraissent utiles lorsqu'on rencontre des énoncés comme (10):

*10. Les générations futures qui seront équipées des outils plus performants

où l'étudiant croyait avoir produit un article indéfini pluriel. On attendrait:

10 '. Les générations futures qui seront équipées $d$ 'outils plus performants

On remarque ainsi que les constructions binominales ne laissent pas toujours deviner la structure verbale directe ou prépositionnelle sous-jacente. C'est pourquoi il est important de faire retrouver aux apprenants les constructions verbales dans des exemples constrastifs:

11. Les générations futures qui utiliseront des outils performants

11 '. Les générations futures qui seront équipées $d$ 'outils performants ${ }^{3}$

${ }^{3}$ Je me souviens encore de la tête d'un étudiant norvégien à qui je tentais d'expliquer qu'il fallait dire être muni d'outils quand on voulait signifier: avoir des outils et être pourvu des outils nécessaires, quand on voulait signifier: avoir les outils nécessaires, et qui pour sortir de l'imbroglio décide de reformuler avec un verbe plus courant: Les générations futures qui utiliseront * d'outils performants pour s'entendre dire sans explication: «Ici il faut des outils quand on veut signifier «avoir des outils»!» 


\section{LES NOMS D'ACTIONS PEUVENT AUSSI REPRÉSENTER DES TYPES}

\section{Lire, c'est de la lutte de classe. (La Mère, Brecht)}

Il faut faire un sort à un préjugé partagé entre enseignés et enseignants selon lequel la nominalisation d'une phrase n'induirait que des structures spécifiantes. Pour Kleiber par exemple, les déverbaux ne sont pas catégorisables. Ils ne se prêtent donc pas à des regroupements en classes. Ils représentent simplement la nominalisation d'un procès avec actants. Or, comme nos exemples le montrent bien, les noms de procès se tiennent en deçà ou au-delà de la frontière de réification. En deçà, ils gardent le dynamisme du verbe et ne présentent pas de structure sous-catégorisante, au delà, le procès peut être associé à un type d'objet et être recatégorisé:

12. J'ai créé beaucoup d'exercices dans ma vie, mais ce type d'activité (hyperonyme du générique: la création d'exercices) n'est pas reconnu institutionnellement.

En (12) l'anaphore conceptuelle n'est pas coréférente au procès verbal, à savoir «la création concrète et répétée d'exercices», et c'est justement la fonction d'opérateur de catégorisation comme: ce genre delce type de, cette sorte de, que de permettre le passage du spécifique au générique [Apothéloz, 1995: 287].

Cette différence pourra être également marquée par le déterminant uniquement, c'est-à-dire par une reprise anaphorique définie ou démonstrative: cette création d'exercices [anaphore coréférentielle] lla création d'exercices [anaphore sans coréférence]

Nom d'action + cpl régime: Avec dét. devant N2 = spécifieur Avec $\varnothing=$ sous-catégorisateur

13. La destruction de l'immeuble «5 rue du Prince» a complètement modifié l'image du quartier

14. Les destructions d'immeubles rapportent plus que leur réhabilitation.

Un truc pratique pour distinguer les deux structures est d'essayer de substituer à N2 l'article possessif, qui est un allomorphe de presque toutes les combinaisons en [DE + art.déf.]. On remarquera que l'article possessif est aussi bien un allomorphe d'un complément générique que d'un complément «singulier».

Tandis que les structures sous-catégorisantes peuvent être paraphrasées par l'hyperonyme [ce type d'activité] ou [ce type d'objet], d’où, pour les exemples précités, respectivement:

13'. Sa destruction a complètement modifié l'image du quartier

14'. Ce type de pratique rapporte plus que la réhabilitation.

Description du processus en texte:

Ce petit dialogue fabriqué nous permet de montrer comment peut évoluer un objet de discours. 
15. - Nous fabriquons un tipi

- Alors comment va la fabrication du tipi?

- La fabrication de tipis demande un tour de main qui ne s'acquiert pas en un jour, et franchement je ne pense pas qu'on puisse faire fortune dans ce genre d'activité.

Il est conseillé de composer, à partir des difficultés des étudiants, des exercices montrant en texte le passage de la spécification à la catégorisation, c'est-à-dire qu'il ne faut pas se contenter d'exercices structuraux consistant à transposer des énoncés propositionnels en syntagmes nominaux complexes, mais il faut imaginer des exercices de structuration textuelle qui manifestent comment un objet de discours évolue «cognitivement», c'est-à-dire dans la représentation de l'énonciateur, au gré de la mise en texte.

On peut aussi relever des exemples de presse et les faire analyser par les étudiants:

16. (a) Le salaire moyen d'un chercheur est de 450.000 roubles ( $110 \mathrm{frs}$ ), il se situe nettement en dessous de la moyenne nationale, si bien qu'on ne peut pas vivre décemment en Russie avec (b) un salaire de chercheur (Presse).

En a) on considère la condition d'un chercheur-type en Russie, dont le salaire est un élément.

En b) ce qui est en cause, ce n'est plus la catégorie «chercheur», mais la catégorie «salaire» qui comprend «le salaire de chercheur» et qu'on évalue mentalement sur une échelle de salaires: salaire de fonctionnaire (de cadre) d'ouvrier etc., pour dire qu'on ne peut vivre avec un salaire de ce genre.

\section{TYPE $\rightarrow$ CATÉGORIE}

17. - Ce matin j'ai été réveillé par le son de clarinette.

- Le son de clarinette gêne moins que celui de basson.

- De toute façon je n'aime pas les instruments pareils, je n'aime que le son de violon et la voix d'homme.

Je n'ai pas pu interroger l'auteur de ce texte ${ }^{4}$, mais comme il avait reçu un enseignement théorique préalable, on peut penser qu'il a interprété les binominaux comme des catégorisateurs. Ce qui est tout à fait logique, sauf pour la première occurrence.

Dans un tel cas le professeur doit avouer que certaines entités ne peuvent pas être sous-catégorisantes. Comme l'a mentionné Berrendonner, [1995: 12-13] «n'importe quel prédicat n'est pas apte à fournir une propriété typique, c'est-à-dire à recevoir une interprétation générique».

Il serait pédagogiquement utile de voir quelles entités ne s'y prềtent pas et pour quelle raison. On peut déjà remarquer que les entités à référent unique ne peuvent être sous-catégorisateurs:

*l'apprentissage de monde

*la lumière de jour

${ }^{4}$ Il s'agit d'un exercice de structuration, qui a été traduit en russe pour les besoins de la cause et proposé à des étudiants russophones en guise de version russe-français. 
Autre remarque: un syntagme indéfini [un N], simple ou complexe, extrait toujours un individu d'une catégorie, si bien que beaucoup de prédicats compléments de N1, peuvent être «typifiés» de manière transitoire: un son de clarinette, un parfum de rose, une odeur de café. Cela ne signifie pas que la catégorie «son de clarinette» existe en langue. Si tel n'est pas le cas, le syntagme [N1DEØN2] ne pourra être construit avec l'article défini à valeur générique ou singulière, *le son de clarinette est divin, *le son de clarinette de Paul. Dans les deux cas, il faut garder l'addition de deux entités: le son de la clarinette.

En résumé: Pour des prédicats génériques, les deux constructions, avec ou sans article intérieur, sont parfois possibles avec les différences signalées en 3 infra; d'autres fois la sous-catégorisation ne s'est pas stabilisée et n'est pas répertoriée dans le lexique. Par exemple on ne catégorise pas les sons en fonction de leur émetteur, mais selon une échelle d'intensité: graves/moyens/aigus. C'est l'adjectif qualificatif qui joue ici le rôle de sous-catégorisateur 5 .

- En (17) on attendrait: j'ai été réveillé par un son de clarinettelle son d'une clarinette, tandis que le son de la clarinette est moins acceptable, car il y a incompatibilité entre le prédicat exprimé par le verbe [ponctuel, transitoire] et la généricité. Une acception singulière [+ connu] est exclue ici.

On dirait en revanche: j'aime le son de la clarinette. Prédicat verbal et nominal expriment tous deux la généralité, ou j'ai été réveillé par la clarinette de Gaspard, prédicat verbal et nominal exprimant conjointement la spécificité.

C'est donc le prédicat verbal qui déterminera la valeur - singulière ou générique - de l'argument.

Le professeur de langue explique généralement qu'on peut dire indifféremment: un son de clarinette ou le son d'une clarinette. Pour Karolak [1995:75], ces deux formes sont permutables: «l'effet final d'indétermination étant le même, on peut rencontrer alternativement dans les mểmes types d'énoncés les formes le $N$ d'un $N$ et un $N$ de $N$ ».

Il en donne pour preuve un exemple tiré de l'Etranger de Camus et sa traduction italienne:

18. Un léger bruit de moteur est monté dans l'air calme jusqu'à nous.

18'. Nell'aria calma è venuto fino a noi il leggero ronzio di un motore.

Cependant selon Karolak, l'usage français et italien semble préférer la structure catégorisante: «la forme la plus largement utilisée en français et en italien est le SN avec l'article indéfini majeur et l'article zéro mineur»

Certes les deux entités des exemples (17) et (18) sont identiques référentiellement, peut-être même sémantiquement, mais pas énonciativement. Un son de clarinette est un filtre plus grossier, il identifie en gros un son, dont la source n'est pas forcément une clarinette (cf. la glose de «chien de bergen»). L'énonciateur pourrait enchaner:

${ }^{5}$ A l'exception de [ØN2] qui est toujours un sous-catégorisateur, tous les autres compléments du nom (adjectifs relationnels, qualificatifs, propositions relatives) peuvent assumer les deux fonctions. 
(...) mais plus tard je me suis rendu compte que c'était le portail du jardin qui grinçait! On pourrait par conséquent dire: «j'adore le son de clarinette de ce portail!» Et dans ce cas il n'y aurait plus identité référentielle. Tandis qu'en identifiant le référent au son d'une clarinette, l'énonciateur a bien pris soin de localiser le son et ceci au cours d'une analyse du type: j'ai été réveillé par un son; c'était le son d'une clarinette. Il serait difficile, voire impossible d'enchaîner avec: ... mais c'était le portail qui grinçait! Il y faudrait au moins des modalisations préalables: $j$ 'ai cru être réveillé par...

\section{LE PHÉNOMÈNE D'HAPLOLOGIE}

On pourrait voir dans l'exemple (14) Les destructions d'immeubles rapportent plus que leur réhabilitation, un pluriel indéfini, sous l'effet de l'haplologie, cette règle édictée en 1647 par Vaugelas dans son Bon Usage et reprise par la grammaire de Port Royal, en1660, qui proscrit, pour des raisons d'euphonie l'article indéfini pluriel, ainsi que les articles partitifs après la préposition [DE].

Cette analyse phonétique est reprise par Nyrop,1914 dans sa grammaire historique de la langue française. Cependant Wilmet [1986: 118], s'appuyant sur Damourette et Pichon, critique cette thèse euphonique, considérant que des répétitions courantes comme certains redoublements de pronoms: nous nous et tu te, qui présentent les mêmes inconvénients, ne choquent pas l'oreille. Wilmet voit dans cette proscription une simplification sémantique. On retiendra de ce débat de grammaire historique qu'une règle de non redoublement neutralise en surface la différence entre sous-catégorisateurs et spécifieurs, lorsque N2 désigne une substance continue avec l'article partitif ou des entités plurielles indéfinies avec l'article [DES]. C'est ainsi que la majorité des quantifiants et des collectifs subissent la loi de l'haplologie, ce qui, pour ces deux importantes catégories de binominaux, simplifie notablement la vie des apprenants. Ainsi dans: Une motte de beurre, un mètre de soie, un panier de prunes, une allée d'arbres.

[DE] est respectivement allomorphe de: [DE du beurre], [DE de la soie], [DE des prunes], [DE des arbres].

Un syntagme comme «des portraits de marquises» pourrait désigner, selon le cotexte, un sous-genre pictural de la catégorie "portrait» ou bien un nombre indéfini d'entités concrètes de la catégorie «marquise».

19a. Le Brun était un peintre secondaire, spécialisé dans les portraits de marquises.

19b. Le chambre du roi était ornée de portraits de marquises, qui furent ses maîtresses".

19c. La chambre du roi était ornée de portraits de marquises, dont certaines furent ses maîtresses.

${ }^{6}$ Certains collègues du colloque ont réagi vivement à (19b), que je me suis empressée de remplacer par (19c). Ils trouvaient inacceptable d'attribuer au concept abstrait «marquises» l'entité concrète «maîtresses du roi» La syllepse serait en effet reprouvée par les puristes. Mais il fallait lire [marquises] comme un pluriel indéfini. Cela démontre aussi que le phénomène d'haplologie n'est pas toujours ressenti. Il est 
Il me semble que, dans ce cas précis, on peut conseiller aux étudiants qui auraient des difficultés à maîtriser cette règle de faire des exercices de transposition sur des énoncés isolés du genre:

20.1. L'ouverture de pistes de luge balisées $=$ des pistes ont été ouvertes

20.2. L'adoption d'enfants $=$ des enfants ont été adoptés

20.3. La disparition d'emplois $=$ des emplois ont disparu

20.4. La perte de temps $=$ du temps a été perdu

En évitant toutefois tout automatisme et en leur faisant à chaque fois décider quelle interprétation du SN sera la plus vraisemblable. Par exemple en (20.1.) le SN complexe joue le rôle de spécifieur à valeur de complément d'objet du prédicat. Un tel prédicat est en effet trop spécifique pour servir de critère de classification. En revanche «piste de luge» entre dans une typologie des pistes, qui comprend des pistes de danse, d'entraînement ... Quant à (20.4.) il est ambigu hors contexte. Il sera plutôt lu comme une nominalisation déverbale avec N2 sujet. Mais dans une mise en contraste énumérative: il passe son temps à déplorer ses pertes: perte d'argent, de temps, d'influence, donc d'amis ... le SN peut fonctionner comme un sous-catégorisateur.

L'activité didactique la plus efficace est de proposer des exercices de repérage dans des articles de journaux, car la prise de conscience de ce phénomème a beaucoup aidé les étudiants dans la compréhension des structures binominales.

Voici quelques exemples d'haplologie tirés de la presse:

26. Après la grève des mineurs, les manifestations d'étudiants l'autre semaine, la gouvernement conservateur a baissé dans les sondages d'opinion. (L'on sait que l'association des étudiants était partagée sur l'opportunité de manifester et que ces manifestations n'ont de loin pas mobilisé tous les étudiants).

27. Révocation de policiers argentins impliqués dans un attentat anti-sémite.

A l'exemple de (27) les titres d'articles de presse sont souvent la version nominale d'un prédicat verbal développé dans le corps de l'article. Un exercice très stimulant est d'essayer de retrouver ces énoncés-sources dans le texte. Ou encore à l'inverse, d'effacer le titre original afin de laisser aux étudiants le soin de trouver des nominalisations résomptives en guise d'intitulés.

Cependant une langue naturelle n'étant pas composée que d'unités discrètes, le scripteur a souvent de la peine à discriminer les deux opérations sémantiques. Cette indécision se manifeste par des variations orthographiques. Voyez cette publicité d'une entreprise de nettoyage, qui d'abord énumère les trois types d'objets de son activité, à la suite de quoi elle se présente comme spécalisée dans un genre particulier de nettoyage: le nettoyage de tuyauterie:

vrai que cet exemple fabriqué est peu coopératif. En effet un scripteur soucieux du confort de son lecteur aurait précisé la valeur du relateur [DE] en évitant l'indéfini [DES] auquel il aurait substitué [PLUSIEURS, QUELQUES], comme le fait, au cours de trois autocorrections, ce journaliste de TV2, commentant les enquètes policières pendant le tour de France: «Il [le directeur sportif] tente d'organiser l'audition des coureurs, de coureurs, de certains coureurs". 


\section{PROTECTION ET NETTOYAGE DE TUYAUTERIES SANITAIRES ET CHAUFFAGES GARANTIE DURANT ET APRĖS TRAVAUX}

\section{DIPAN \\ PROTECTION ET NETTOYAGE DE TUYAUTERIE}

Une telle ambiguitté se manifeste également dans la traduction d'une langue latine à une autre. Voici encore un exemple italien-français. Il s'agit du fameux film de Dino Risi «Profumo di donna «, que le français traduit en «Parfum de femmes», où l'on ressent une multitude vague d'individus: il y a des femmes dans l'air et je perçois leur parfum se se dit le héros. Tandis que l'italien extrait de cette multitude hétérogène un nouveau type de parfum ${ }^{7}$.

\section{ERREURS TRIBUTAIRES DE RÈGLES FAUTIVES}

\section{9. *J'ai la leçon de français}

Premier préjugé à combattre: «Le nom-tête est précédé de l'article défini s'il a une expansion». Généralisation de la règle: $\mathrm{SN}=$ prédicat simple $\rightarrow$ [art. indéfini $+\mathrm{N}$ ] [j'ai trouvé un chat] $/ \mathrm{SN}=$ prédicat complexe $\rightarrow$ [art. défini $+\mathrm{N}$ ] [j'ai trouvé le chat du voisin].

L'étudiant généralise une règle qui traîne dans plusieurs méthodes de français: Il motive ainsi son erreur: «le nom simple demande l'indéfini quand on introduit un objet nouveau, mais on rétablit le défini quand le premier nom est accompagné d'un complément». Or cette règle, comme l'ont bien démontré les travaux de Stanisław Karolak, n'est valide que lorsque le complément de nom est extensionnel et représente un deuxième argument qui vient saturer le nom-tête, c'est-à-dire lorsqu'on a affaire à un spécifieur, en (29) nous avons un seul objet complexe, désignant une catégorie de leçon. J'ai [une, ma] leçon de français.

A noter que «la leçon de français» serait possible sous certaines conditions contextuelles ou de savoir partagé: aujourd'hui j'ai la leçon de français et non pas celle de mathématiques. Il serait plus naturel de dire: «aujourd'hui j'ai le français et pas les math».

\footnotetext{
7 Voilà ce que dit Korzen (1996) travaillant sur l'italien.

Les syntagmes SNØ conceptuels apparaissent au singulier et au pluriel: au singulier ils dénotent une catégorialité abstraite de masse homogène s'il s'agit de noms [- numérable] ou de type unifié, homogénéisé d'individus hétérogènes avec des noms [+ numérable] et expriment en soi le trait [- numérable]. Au pluriel ils dénotent une catégorie abstraite d'entités hétérogènes et distinctes (individus ou sous-types) et le trait [+ numérable].

Dans certains cotextes le SN $\varnothing$ au pluriel avec des noms [+ numérable] permet aussi de désigner un nombre délimité d'entités d'une catégorie, c'est-à-dire un trait sémantique d'extensité. Une telle lecture est favorisée par d'autres éléments contextuels de spécification, par exemple des attributs, des localisations spatio-temporelles, dans ces cas les SN $\emptyset$ au pluriel ont - comme les noms accompagnés d'un article - une plus grande liberté de manifestation. Traduit par nos soins.
} 
Deuxième préjugé, en contradiction avec le précédent:

30. *Les cours de la linguistique

31. *Les graffitis servent à marquer un territoire d'un groupe

Les déterminants des binominaux bougent ensemble selon la combinaison: «[UN N1 DE ØN2] [un cours de linguistique] vs [LE N1 DU N2] [*les cours de la linguistique] ou encore deux indéfinis vs deux définis, [le territoire du groupe] vs [*un territoire d'un groupe]». Or, comme nous l'avons vu infra, si le binominal désigne un type, la valeur notionnelle de N2 sera toujours désignée par $\emptyset$, et ne subira pas l'influence de saisie de $\mathrm{Nl}$. C'est ce que montre l'échange authentique (32):

32 a) - J'exige un droit de regard sur l'avancement du travail.

32 b) - Le droit de regard n'est prévu que pour la direction du projet

- Un [N2indéf.] requiert aussi le défini devant N1 car le SN ainsi formé représente toujours deux objets de pensée, peu importe que le nom-tễte soit défini par un N2objet extensionnel déterminé ou par un spécimen indéterminé d'une autre catégorie. On dira alors que le référent est identifié mais pas déterminé. La forme substitutive serait également l'article possessif: [le cri de l'enfant vs d'un enfant $\rightarrow$ son cri]

\section{Troisième préjugé:}

\section{3. * Le mécontentement de population}

«Un adjectif épithète relationnel correspond toujours à un nom sous-catégorisateur. D'après les explications de l'auteur de cet exemple, celle-ci aurait d'abord écrit la forme correcte: «le mécontentement de la population» mais, conformément à une notion grammaticale mal comprise, s'lon laquelle un complément en [ØN] a valeur d'adjectif, elle a appliqué le test de la substitution: «mécontentement populaire», qui lui a semblé l'indice déterminant pour voir dans l'adnominal un catégorisateur. D'autres étudiants reconnaissent aussi dans ce procédé un truc pratique qu'on leur a enseigné dans leur pays. Ce qui donne sous leurs plumes: «le problème de l'alcoolisme doit être résolu par des mesures de gouvernement». Or l'adjectif relationnel joue les deux rôles et représente selon les contextes tantôt un catégorisateur, tantôt un spécifieur, cf. note 5. Parfois il est exclusivement spécifieur, les soins corporels $=$ les soins $d u$ corps, où le syntagme représente un type de soins (*ses soins).

Le préjugé de l'adjectif sous-catégorisant est si bien ancré, même chez des enseignants de français, qu'il me paraît utile de montrer par quelques exemples, tous tirés de la presse, que l'adjectif assume très souvent le rôle de spécifieur:

34. Il a été porté par l'enthousiasme collectif

35. Pris de court par cette mobilisation citoyenne, le gouvernement semble reculer.

36. Le partage vocalique et consonantique.

37. Le président russe a (...) écarté une proposition parlementaire visant à amender la Constitution pour confier un éventuel interim de la présidence du pays au président de la chambre haute (presse).

38. Marcha Berry, très au fait de la garde-robe présidentielle, nous tient au parfum: le tailleur bleu ciel qu'a revêtu la First Lady est signé Oscar de la Renta. 
Quand j'engage les étudiants à donner la préférence, quand ils ont à choisir un complément de $\mathrm{N} 1$, à un adjectif, parce qu'il neutralise la différence entre spécifieur et catégorisateur, ils me répondent qu'on leur a toujours conseillé d'ěviter les adjectifs, «à cause de leur instabilité sémantique», les encourageant, au contraire, à leur substituer un nom. Les deux exemples suivants montrent que ce conseil est bon!

*39. Il suffit de créer une émission informative

*40. La politique austère menée par les Anglais

\section{CONCLUSION}

J'ai tenté de montrer tout ce qu'apporte l'analyse des erreurs à la didactique du français langue étrangère. On l'aura compris, l'enseignant ne saurait se limiter à corriger quelques déterminants, puisque, par le biais d'un déterminant, c'est la vision énonciative qui change!

L'analyse des erreurs prend au sérieux la capacité cognitive de l'étudiant, le gratifiant d'erreurs motivées. Les étudiants ne s'y trompent d'ailleurs pas et apprécient beaucoup ces ateliers de réparation de leurs copies, à tel point que lorsqu'ils ne retrouvent pas leurs productions dans la typologie des erreurs proposée au groupe, ils protestent qu'on les a oubliés.

L'analyse des erreurs conduit également l'enseignant à interroger les descriptions de la linguistique fondamentale et parfois à les remettre en cause. Car ce qu'il recherche, c'est une théorisation «unifiante», qui considère le fonctionnement de tout le système. Beaucoup de modèles sémantiques de haut niveau nous amènent à la pulvérisation des descriptions et aux aléas de la mémorisation. Certes avec un modèle unifié - la langue n'étant pas un système mathématique - les étudiants feront encore des erreurs, mais qui mettront en cause, cette fois, les failles du système linguistique: «le son de clarinette» est une de ces erreurs!

\section{BIBLIOGRAPHIE}

A pothéloz, D. (1995): Rôle et fonctionnement de l'anaphore dans la dynamique textuelle, Genève, Librairie Droz.

B ally, C. (1965): Linguistique générale et linguistique française, Berne, Francke, (4e éd. revue et corrigée).

B en etti, L. (1995): Typologie des syntagmes binominaux de type «NI de N2» recueillis dans des manuels homéopathiques, Tranel 23, décembre 1995, 57-76.

Berrendonner, A. (1995): Quelques notions utiles à la sémantique des descripteurs nominaux, Tranel 23, décembre 1995, 9-39.

Giusti, F. (1981): La referenza nominale in una lingua senza articolo. Analisi comparativa del russo e dell'italiano, Studi di Grammatica italiana, Vol X, Accademia della Crusca, Firenze.

Grevisse, M. (1993): Le bon usage, grammaire française refondue par André Goosse. Duculot. 
Karolak, S. (1989): L'article et la valeur du syntagme nominal, Paris, Presses Universitaires de France.

Korzen, I. (1996): L'articolo italiano fra concetto ed entità, Etudes Romanes 36, Institut d'Etudes Romanes, Université de Copenhague.

Mondada, L. (1994): Verbalisation de l'espace et fabrication du savoir, Approche linguistique de la construction des objets de discours, thèse de doctorat présentée à la faculté des lettres de Lausanne.

Nowakowska, M. (1993): Les problèmes de structure immanente dans le syntagme nominal abstrait complexe en français et en italien, Cracovie, Wydawnictwo Naukowe WSP (Etudes monographiques de l'Ecole Normale Supérieure à Cracovie, $\mathrm{Nr}$ 174).

Riegel, M., Pellat, J-C., Rioul, R. (1994): Grammaire méthodique du français, Paris, P.U.F. W ilmet, M. (1986): La détermination nominale, P.U.F. linguistique nouvelle. 